ANÁLISE DA ATIVIDADE ANTIOXIDANTE DE DIFERENTES EXTRATOS FOLIARES DE RUTA GRAVEOLENS

\title{
ANALYSIS OF ANTIOXIDANT ACTIVITY OF DIFFERENT LEAF EXTRACTS OF RUTA GRAVEOLENS
}

\author{
SANTOS, Vanessa Freitas ${ }^{1 *}$; ALVES, Blyeny Hatalita Pereira ${ }^{2}$ \\ 1,2 Instituto Federal de Educação, Ciência e Tecnologia de Goiás, Câmpus Itumbiara, Av Furnas, 55, cep 75524- \\ 010, Itumbiara - GO, Brasil \\ (fone: +55 64 21035600) \\ ${ }^{*}$ Autor correspondente \\ e-mail: lab.cienciasifg@gmail.com
}

Received 23 July 2014; received in revised form 31 July 2014; accepted 5 August 2014

\section{RESUMO}

O interesse pelo estudo dos produtos naturais cresce sistematicamente em todo o mundo. No Brasil isso ocorre devido à grande quantidade de espécies da flora brasileira que são utilizadas popularmente na medicina alternativa, culinária, e até mesmo como cosméticos. No entanto, devido à grande diversidade, ainda são poucas as que foram alvos de estudos mais sistemáticos pelas comunidades científicas. A Ruta graveolens (arruda) é uma planta conhecida em diversas regiões do país por apresentar atividade analgésica, antihemorrágica e calmante quando ingerido o chá das folhas e abortiva quando o suco das folhas é misturada com alho e cominho. A infusão das folhas é usada contra cólicas menstruais, diarreia, febre, e o xarope das folhas usado contra tosses graves ( Di Stasi e Hiruma- Lima 2002). Porém, poucas pesquisas foram realizadas com intuito de analisar sua composição química e sua atividade antioxidante. Neste sentido, esta pesquisa consistiu em analisar a atividade antioxidante dos extratos foliares de Ruta graveolens. As extrações foram realizadas pelos métodos de infusão das folhas secas e através do extrator de lipídeos com folhas frescas e secas com os solventes hexano, álcool e água. A atividade antioxidante foi determinada pelo radical livre estável 2,2-difenil-1-picril-hidrazil (DPPH') seguindo o método descrito por Lima (2008). Os extratos aquosos atingiram melhor rendimento em relação às extrações com os solventes hexano e álcool e as folhas frescas apresentaram um rendimento inferior às folhas secas. A espécie apresentou atividades antioxidantes confirmadas através dos cálculos de $\mathrm{CE}_{50}$, o extrato aquoso de folha seca apresentou a maior atividade antioxidante, inibindo $78,98 \%$ dos radicais $\mathrm{DPPH}^{\circ}$.

Palavras-chave: Química dos Produtos Naturais, Plantas curativas, radicais livres, 2,2-difenil-1-picril-hidrazil (DPPH)

\section{ABSTRACT}

The interest in the study of natural products has grown steadily worldwide. In Brazil, it has occurred due to the large number of species of flora which are popularly used in alternative medicine cuisine, and even as cosmetics. However, due to big diversity, there are few that have been targets of more systematic studies by the scientific communities. The Ruta graveolens (rue) is known in various regions of the country by presenting analgesic, antihemorrhagic and soothing activity when ingested tea leaves and abortive when the juice of the leaves is mixed with garlic and cumin plant. The infusion of the leaves is used against menstrual cramps, diarrhea, fever, and syrup from the leaves used against severe coughs (Di Stasi and Hiruma-Lima 2002). However, few researches have been conducted with a view to analyzing their chemical composition and their antioxidant activity. In this sense, this research was to examine the antioxidant activity of leaf extracts of Ruta graveolens. The extractions were performed by the methods of infusion of dried leaves and through the extractor lipids with fresh and dried leaves with hexane solvents, alcohol and water. The antioxidant activity was

PERIÓDICO TCHÊ QUÍMICA • www.periodico.tchequimica.com • Vol. 12 N. 23.

• ISSN 1806-0374 (impresso) • ISSN 1806-9827 (CD-ROM) • ISSN 2179-0302 (meio eletrônico)

(C) 2015. Porto Alegre, RS. Brasil 
determined by 2,2-diphenyl-1-picryl-hydrazyl stable free radical $\left(\mathrm{DPPH}^{\circ}\right)$ following the method described by Lima (2008). The aqueous extracts achieved better performance compared to extraction with hexane solvents and alcohol and fresh leaves have presented a lower yield than dried leaves. The specie has presented antioxidant activities confirmed through calculations of EC50, the aqueous extract of dried leaf has showed the highest antioxidant activity, inhibiting $78.98 \%$ of $\mathrm{DPPH}^{\bullet}$ radicals.

Keywords: Chemistry of Natural Products, healing plants, free radical, 2,2-diphenyl-1-picryl-hydrazyl (DPPH')

\section{INTRODUÇÃO}

Atualmente, ainda é possível perceber, com relativa frequência o uso de plantas no tratamento de doenças. Essa prática, eficaz ou não, é tradicional e utilizada há milhares de anos. Em busca de melhores condições de vida, o homem necessitou recorrer à natureza para obter fontes de alimentação e proteção à saúde e a partir de suas observações e experiências, sofreu um processo biológico evolutivo descobrindo, nas plantas, o tratamento de injurias e doenças (MIGUEL, 1999).

Esses conhecimentos ancestrais e que foram repassados através de gerações são, em grande parte, empíricos. Pouco se conhece sobre as suas composições e os efeitos químicos e biológicos em seres vivos. Segundo Cechinel Filho e Yunes (1998), a vegetação mundial contém cerca de 500 mil espécies de plantas, dentre as quais somente $5 \%$ são estudadas por comunidades científicas. Esses dados revelam a necessidade de investir cada vez mais em pesquisas voltadas para a área de produtos naturais, já que, de acordo com os autores supracitados, $80 \%$ da população utilizam basicamente medicamentos de origem vegetal, devido ao seu baixo custo.

Além das propriedades medicinais, os compostos extraídos das plantas permitem uma ampla aplicação em indústrias agrícolas, cosméticas e alimentícias. No uso agrícola, o estudo é voltado às ações alelopáticas e à extração de substâncias usadas no controle de pragas. Nas indústrias cosméticas e alimentícias, o interesse é pela busca de nutrientes importantes para o corpo humano, em especial compostos que possuam ação antioxidante, pois estes apresentam efeitos benéficos, principalmente contra o envelhecimento precoce (LIMA, 2008).

Este trabalho focalizou o estudo e a análise da atividade antioxidante de uma planta medicinal originária da Europa Meridional e cultivada em vários países, como o Brasil, e especialmente da Europa Oriental: Ruta graveolens L., conhecida popularmente por sua versatilidade, é usada por apresentar propriedades fitoterápicas e condimentares.

Apesar da espécie em questão ser bastante conhecida e utilizada no país, uma busca bibliográfica realizada em sites de pesquisa com as palavras- chaves: arruda, Ruta graveolens, composição química da Ruta graveolens e atividade antioxidante de Ruta graveolens, indicam que até 0 presente momento, poucos estudos científicos referentes aos constituintes químicos e a potencial ação antioxidante da planta são encontrados na literatura. Com isso, o objetivo deste trabalho foi Avaliar a atividade antioxidante dos extratos foliares de Ruta graveolens (arruda).

\section{METODOLOGIA DA PESQUISA}

\subsection{Coleta da espécie}

As amostras de folhas foram coletadas em hortas e residências no município de Itumbiara - GO, respectivamente nos bairros Vila Vitoria I e Santos Dumont. As coletas foram realizadas nos meses de Setembro de 2011, Janeiro e Fevereiro de 2012. Todas as coletas foram realizadas no período da manhã.

\subsection{Análise Química}

As análises químicas foram realizadas no IFG-Campus Itumbiara e no laboratório de Produtos Naturais do Instituto de Química da Universidade Federal de Uberlândia. No IFG, foram realizadas as extrações e no laboratório de Produtos Naturais do Instituto de Química da Universidade Federal de Uberlândia foram realizados os testes de atividade antioxidante. 


\subsection{Preparo das amostras}

A coleta das folhas e preparo das amostras iniciou-se no mês de Setembro. Imediatamente Após a coleta, as folhas foram embaladas em sacos plásticos que foram lacrados e levados ao Laboratório de Química do IFG-Campus Itumbiara e colocadas para secar em uma estufa à temperatura de $36^{\circ} \mathrm{C}$ e deixadas por cerca de $76 \mathrm{~h}$. Após isso, foram trituradas, pesadas e condicionadas em béqueres plásticos vedados com parafilme em freezer e utilizadas gradativamente para as extrações.

Posteriormente coletou-se mais folhas nos meses de janeiro e fevereiro de 2012 foram lacradas em sacos plásticos e levadas ao Laboratório do IFG-Campus Itumbiara, onde foram cortadas, pesadas e de imediato levadas para extração.

\subsection{Determinação de umidade}

Para determinação de umidade, colocouse três amostras de folhas em uma estufa por aproximadamente $24 \mathrm{~h}$ a uma temperatura de $105,0^{\circ} \mathrm{C}$ (Estufa de esterilização e secagemDL-SE). Após esse tempo, as amostras foram pesadas e calculou-se a umidade por diferença de peso (massa inicial - massa final). $O$ teor de umidade foi expresso em \%.

\subsection{Extração}

\subsubsection{Extração por extrator de lipídeos}

Em um primeiro momento, foram pesadas cerca de $5 \mathrm{~g}$ da folha seca, em uma balança analítica e colocada em um cartucho de celulose próprio para este método. $\mathrm{O}$ cartucho juntamente com a amostra foi inserido em um extrator de lipídeos da Solab Cientifica, e a extração foi realizada com três (03) solventes, hexano, etanol e água, em sequência. Depois, a extração foi realizada com aproximadamente $5 \mathrm{~g}$ de folhas frescas do mesmo modo da primeira. O tempo necessário para cada extração foi de aproximadamente 9 horas. $O$ extrato recolhido foi separado do solvente por rotoevaporação, condicionado em frascos com tampa e armazenados em freezer até a utilização.

\subsubsection{Extração por Infusão.}

Para extração por infusão, foram colocados em um béquer $100 \mathrm{~g}$ de folhas secas com $250 \mathrm{~mL}$ de água destilada e em outro a mesma quantidade de folhas em $250 \mathrm{~mL}$ de etanol e tampadas com vidro de relógio vedado por parafilme, essa mistura foi deixada em extração por sete dias e então foi filtrada e rotoevaporada para separação do solvente.

\subsection{Atividade antioxidante e cálculo de $\mathrm{CE}_{50}$ (concentração de inibição de $\mathbf{5 0} \%$ do DPPH)}

A atividade antioxidante foi determinada através do radical livre estável 2,2-di(4-toctilfenil)-1-picrihidrazila $\left(\mathrm{DPPH}^{\circ}\right)$ seguindo 0 método descrito por Lima (2008). Preparou-se uma solução de $\mathrm{DPPH}^{\circ}, 40 \mu \mathrm{g} / \mathrm{mL}$ em metanol, construindo-se uma curva analítica com diferentes concentrações $(40,35,30,25,20,15$, 10, $5 \mu \mathrm{g} / \mathrm{mL}$ ).

Para quantificação dos extrativos solúveis, $1,00 \mathrm{~mL}$ de cada extrato foi recolhido e seco num frasco calibrado a $105^{\circ} \mathrm{C}$, durante 6 horas em estufa e depois, resfriado à temperatura ambiente em dessecador e pesado. Os extratos foram diluídos em metanol nas concentrações de $600,480,360,240,120$ e 60 $\mu \mathrm{g} / \mathrm{mL}$. Para avaliar a atividade antioxidante foram colocados $0,3 \mathrm{~mL}$ de cada concentração das amostras e $2,7 \mathrm{~mL}$ da solução previamente preparada de $\mathrm{DPPH}^{\circ}$ em tubos de ensaio em duplicata. Foi feito um branco nas mesmas condições, com todos os reagentes, exceto a amostra. Posteriormente foram feitas as leituras a cada 15 minutos durante 1 hora no comprimento de onda de $517 \mathrm{~nm}$, em um espectrofotômetro Shimatzu UV- 1601 PC.

Seguindo ainda a metodologia de Lima (2008), para obtenção do $\mathrm{CE}_{50}$ (concentração de inibição de $50 \%$ do $\mathrm{DPPH}^{\circ}$ ), calculou-se a porcentagem de $\mathrm{DPPH}^{\circ}$ remanescente, empregando a equação 1 :

\section{$\% \mathrm{DPPH}^{\circ} \mathrm{rem}=\left\{\left[\mathrm{DPPH}^{*}\right] \mathrm{am} /\left[\mathrm{DPPH} \mathrm{H}^{\circ}\right] \mathrm{bran}\right\}$ * 100}

Em que: [DPPH'] am: concentração de DPPH' na concentração avaliada (extrato), [DPPH']bran: concentração de $\mathrm{DPPH}^{\circ}$ no branco (todos os reagentes exceto a amostra) 
Para obter-se a porcentagem de DPPH ${ }^{\circ}$ inibido pelas amostras, utilizou-se a equação 2 :

$\% \mathrm{I}=100-\% \mathrm{DPPH}^{\circ} \mathrm{REM}$

E para obtenção da $\mathrm{CE}_{50}$, plotaram-se os valores de \% $\mathrm{DPPH}^{*}$ versus as concentrações das amostras analisadas.

\section{RESULTADOS E DISCUSSÃO:}

\subsection{Determinação de umidade}

A determinação de umidade foi feita por diferença de peso. As folhas de arruda apresentaram um teor de umidade, em porcentagem, de $72,50 \pm 0,59 \%$.

\subsection{Extrações}

\subsubsection{Extração por extrator de lipídeos}

Nas extrações pelo extrator de lipídeos foram obtidos os extratos hexanólico, etanólico e aquoso das folhas secas e frescas de Ruta graveolens. Na extração das folhas frescas com hexano, não foi possível quantificar os extrativos, devido ao alto teor de umidade. Pois segundo Dias et al.(2004) a secagem afeta sobremaneira o rendimento e a composição química das espécies, especialmente as aromáticas por possuírem substâncias muito voláteis, que são facilmente perdidas devido à variação de temperatura. A secagem das plantas minimiza a perda de princípios ativos e retarda a deterioração em decorrência da redução da atividade enzimática, permitindo a conservação das plantas por um período maior e um melhor rendimento extrativo (COSTA et.al, 2005).

\subsubsection{Infusão}

A infusão etanólica e aquosa foi realizada com as folhas secas devido à conservação pela atuação regressiva na ação enzimática e a maior quantidade de princípios ativos em relação à folha fresca (COSTA et.al, 2005). Os rendimentos dos extrativos por infusão foram inferiores a extração por extrator de lipídeos conforme é apresentado na tabela 1.

\subsection{Atividade antioxidante e cálculo de $\mathrm{CE}_{50}$ (concentração de inibição de $50 \%$ do DPPH)}

A atividade antioxidante foi calculada pela inibição do radical $\mathrm{DPPH}^{\bullet}$, que segundo Lima (2008), mede a capacidade de consumo do radical livre (1,1- Difenil-2- picrilidrazila), de coloração púrpura, formando um radical estável e incolor pelo recebimento de átomos de hidrogênio radicalar e elétrons.

Para a análise da atividade antioxidante, foi construída uma curva de calibração do DPPH ${ }^{\circ}$ (Figura 1), que permite determinar a concentração desse radical em função do tempo da reação. Essas concentrações foram usadas para o cálculo da \% de inibição do radical $\mathrm{DPPH}^{\circ}$.

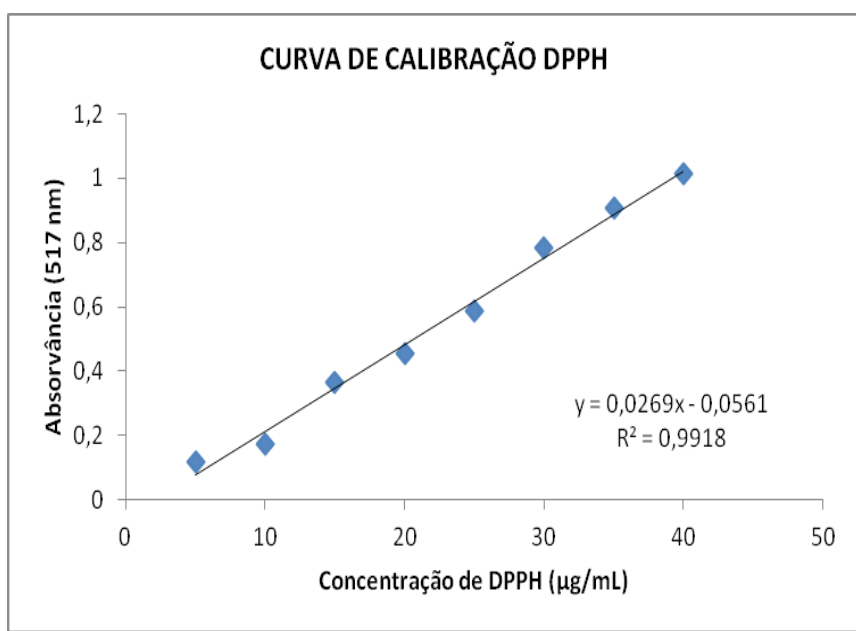

FIGURA 1- Curva de Calibração do DPPH'

Os extratos analisados foram diluídos em metanol em concentrações de 600, 480, 360, 240, 120 e $60 \mu \mathrm{g} / \mathrm{mL}$ e destas soluções foram retirados $0,3 \mathrm{~mL}$ de cada concentração, em duplicata, para reação com DPPH'.

As soluções foram cobertas por papel alumínio e manuseadas em baixa iluminação para evitar a fotodegradação do $\mathrm{DPPH}^{*}$ e a cada 15 minutos eram submetidas à leitura no espectrofotômetro por 1 hora, conforme método utilizado por Lima (2008).

A figura 2, mostra a variação da coloração do $\mathrm{DPPH}^{\circ}$, cada cor representa a intensidade da reação e consequentemente a atividade antioxidante em cada concentração.

A solução de $\mathrm{DPPH}^{{ }^{*}}$ originalmente apresenta uma coloração violeta e quando em contato com uma substância antioxidante, que consegue sequestrar o radical DPPH ${ }^{\circ}$, este muda a coloração para um tom amarelado (DUARTEALMEIDA, 2006). 


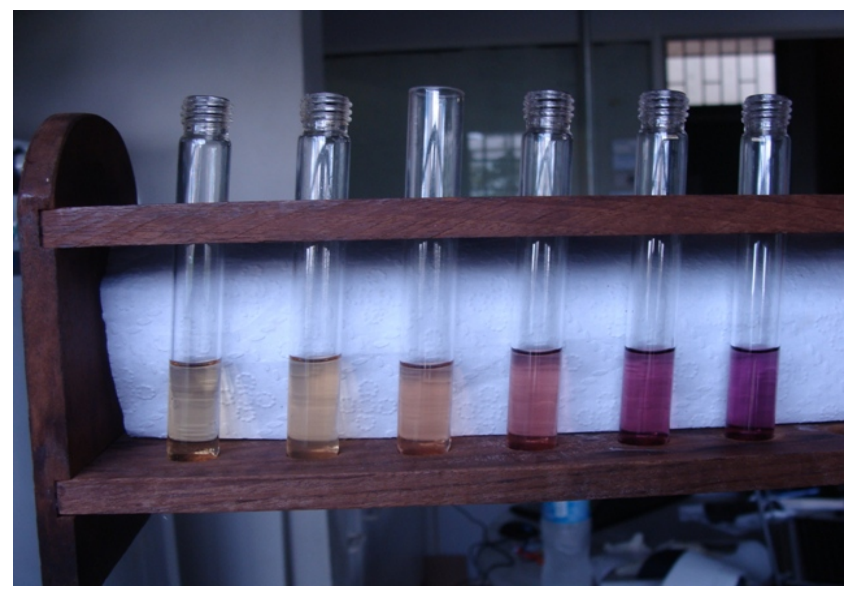

FIGURA 2- Reatividade do radical $D P P H^{\circ}$ com diferentes concentrações de extrato aquoso de folhas secas de Ruta graveolens

Os resultados obtidos de cada amostra, pelas absorvâncias em cada tempo seguem na tabela 2.

As equações utilizadas para o cálculo da porcentagem de $\mathrm{DPPH}^{\circ}$ remanescente, porcentagem inibitória de $\mathrm{DPPH}^{\bullet}$ e concentração de inibição de $50 \%$ do $\operatorname{DPPH}^{*}\left(\mathrm{CE}_{50}\right)$, estão expressas na metodologia, e foram respectivamente as Equações 1 e 2.

$A E_{50}$ é o indicador de presença ou não de atividade antioxidante na amostra, de acordo com Chang et al. (2001), quanto maior o consumo de $\mathrm{DPPH}^{\circ}$ por uma amostra, menor será a sua $\mathrm{CE}_{50}$ (concentração de inibição de $50 \%$ do $\mathrm{DPPH}^{\circ}$ ) e maior a sua atividade antioxidante.

Os valores demonstrados na tabela anterior indicam que o extrato aquoso da folha seca apresentou $\mathrm{O}$ menor valor de $\mathrm{CE}_{50}$, apresentando a maior atividade antioxidante dentre os extratos analisados. Os extratos aquosos apresentaram maior atividade antioxidante que os extratos etanólicos, o mesmo foi observado para os extratos de folhas secas em comparação com as frescas.

A elevada atividade antioxidante das folhas secas, em detrimento às folhas frescas, pode ser explicada pelo melhor rendimento dos extratos, devido à redução da degradação enzimática e a consequente minimização das perdas de princípios ativos (COSTA et al, 2005).

Foram feitas comparações com outras plantas que tiveram os efeitos antioxidantes testados. Dentre as plantas apresentadas, o extrato alcoólico (folha fresca e seca) e o extrato hexano (folha seca) de Ruta graveolens apresentaram atividade antioxidante bastante inferior em relação às outras, já o extrato aquoso (folha seca) demonstrou uma boa atividade antioxidante sendo superior em relação à Eugenia uniflora $L$ conforme apresentado na Tabela 3.

\section{CONCLUSÕES}

Verificou-se que as extrações aquosas atingiram um melhor rendimento de extratos em relação aos solventes etanol e hexano, devido a interação polar do grupo carbonila, presente nas cetonas, componentes majoritários da Ruta graveolens.

Também foi possível analisar que as folhas frescas apresentaram um rendimento inferior e consequentemente menor atividade antioxidante, devido ao alto teor de umidade, que favorece a perda de princípios ativos pela volatilização devido à variação de temperatura.

A espécie apresentou atividades antioxidantes confirmadas através dos cálculos de $\mathrm{CE}_{50}$ e comparadas com outras espécies já analisadas. $O$ extrato aquoso da folha seca foi o que melhor solubilizou substâncias com princípios antioxidantes, apresentando o menor valor de concentração de inibição de $50 \%$ de $\mathrm{DPPH}^{\circ}$.

Outros estudos poderão ser realizados com óleos essenciais e extratos desta planta, a fim de comparar a atividade antioxidante e analisar as possíveis atividades fungicidas e antimicrobianas por diferentes métodos.

\section{AGRADECIMENTOS}

Ao Instituto Federal de Educação, Ciência e Tecnologia de Goiás, Campus Itumbiara pelo financiamento da pesquisa. Ao laboratório de Química dos Produtos Naturais da Universidade Federal de Uberlândia. 


\section{REFERÊNCIAS}

1. Chang, S.T., Wu, J.H., Wang, S.Y., Kang, P.L., Yang, N.S., Shyur, L.F. Antioxidant activity of extracts from Acacia confusa bark and heartwood. J. Agric. Food Chem., v. 49, p. 3420-3424, 2001.

2. Costa, L.C.B., Corrêa, R.M., Cardoso, J.C.W., Pinto, J.E.B.P., Bertolucci, S.K.V., Ferri, P.H. Secagem e fragmentação da matéria seca no rendimento $e$ composição do óleo essencial de capimlimão. Horticultura Brasileira, Brasília, v.23, n.4, p.956-959, 2005.

3. DI STASI, L.C., HIRUMA-LIMA, C.A. Plantas medicinais na Amazônia e na Mata Atlântica. São Paulo: Editora UNESP, 2002.

4. Dias, A.G; COSTA, M.A., GUIMARÃES, I.C. Guia prático de química orgânica técnicas e procedimentos: aprendendo a fazer. Rio de Janeiro: Interciência, 2004.

5. DUARTE-ALMEIDA, J.M., SANTOS, R.J., GENOVESE, M.I., LAJOLO, F.M. Avaliação da atividade antioxidante utilizando sistema $\beta$-caroteno/ácido linoleico e métodos de sequestro de radicais $\mathrm{DPPH}$. Ciência, tecnologia. E alimentos, Campinas, v.26, n.2, p. 446452, 2006.

6. CECHINE FILHO, V., YUNES, R.A. Estratégia para a obtenção de compostos farmacologicamente ativos a partir de plantas medicinais. Conceitos sobre modificação estrutural para otimização da atividade. Química Nova, v.21, n. 1, 1998.

7. LIMA, R.K. Óleos essenciais de Myristica fragrans Houtt. e de Salvia microphylla H. B. K.: caracterização química, atividade biológica e antioxidante. Tese (Doutorado) Programa de Pós- graduação em Agroquímica, Universidade Federal de Lavras, Lavras - MG, 2008.

8. MIGUEL, M.D., MIGUEL, O.G. Desenvolvimento de fitoterápicos. São Paulo: Probe Editorial,1999.
9. NUNES, X.P., MESQUITA, R.F., SILVA, D.A., LIRA, D.P., COSTA, V.C.O., SILVA, M.V.B., XAVIER, A.L., DINIZ, M.F.F.M., AGRA, M.F. Constituintes químicos, avaliação das atividades citotóxica e antioxidante de Mimosa paraibana Barneby (Mimosaceae). Revista brasileira de farmacognosia. João Pessoa, v.18, 2008.

10. RIBEIRO, S.R., FORTES, C.C., OLIVEIRA, S.C.C., CASTRO, C.F.S. Avaliação da atividade antioxidante de solanum paniculatum (solanaceae). Arq. Ciênc. Saúde Unipar. Umuarama, v. 11, n. 3, p. 179-183, 2007.

11. SILVA , F.G.E., MENDES , F.R.S., CAVALCANTE, F.L., BARBOSA, F.G., ASSUNÇÃO, J.C.C. Composição Química e atividade nematicida do óleo essencial de Ruta graveolens (arruda). V CONNEPI, 2010. Instituto Federal de Educação, Ciência e Tecnologia do Ceara. Quixada, 2010.

12. SILVA, J.K.R., GIRARD, C.K.T., ANDRADE, E.H.A; GUIMARÃES, E.F; MAIA, J.G. Polifenóis totais e capacidade antioxidante de extratos de espécies de Piper (Piperaceae) com ocorrência na Serra de Carajás, PA. $32^{\circ}$ encontro anual da sociedade brasileira de Química. Fortaleza, 2009. Disponível em: http://sec.sbq.org.br/cdrom/32ra/resumos/ T1259-1.pdf. Acesso em: 09/04/2012.

13. TORIANE, A.L.T., OLIVEIRA, L. Ruta graveolens $\mathrm{L}$.: $\mathbf{O}$ conhecimento e suas particularidades. 2006. 70p. Monografia de especialização- Faculdades Integradas "espitira". Curitiba, 2006.

14. VICENTINO, A.R.R., MENEZES, F.S. Atividade antioxidante de tinturas vegetais, vendidas em farmácias com manipulação e indicadas para diversos tipos de doenças pela metodologia do DPPH. Revista brasileira de farmacognosia. João Pessoa, v.17, 2007.

15. VICTORIA, F.N., SAVEGNEGO, L., LENARDÃO, E.J. Atividade antioxidante in vitro do óleo essencial de Pitanga. XIII ENPOS. Londrina, 2011. Disponível em: http://www.ufpel.edu.br/enpos/2011/anais/ 
pdf/CB/CB_00360.pdf. Acesso em:

09/04/2012.

Tabela 1- Quantificação dos extratos de Ruta graveolens para cada extração realizada

\begin{tabular}{l|l}
\hline Extratos de Ruta Graveolens & Rendimento \\
\hline 1 Extrato hexano- folha seca & $670 \mu \mathrm{g} / \mathrm{mL}$ \\
2 Extrato etanólico- folha seca & $1260 \mu \mathrm{g} / \mathrm{mL}$ \\
3 Extrato aquoso- folha seca & $3000 \mu \mathrm{g} / \mathrm{mL}$ \\
4 Extrato etanólico - folha fresca & $800 \mu \mathrm{g} / \mathrm{mL}$ \\
5 Extrato aquoso- folha fresca & $4570 \mu \mathrm{g} / \mathrm{mL}$ \\
6 Infusão alcoólica & $13170 \mu \mathrm{g} / \mathrm{mL}$ \\
7 Infusão aquosa & $19833 \mu \mathrm{g} / \mathrm{mL}$ \\
\hline
\end{tabular}

Tabela 2- Valores da \% DPPH remanescente, porcentagem de inibição e concentração inibitória de $50 \%$ dos radicais $\mathrm{DPPH}$

\begin{tabular}{|l|c|c|c|}
\hline Amostras & $\begin{array}{l}\text { \% DPPH } \\
\text { remanescente }\end{array}$ & $\begin{array}{l}\text { \% de } \\
\text { inibição }\end{array}$ & $\begin{array}{l}\text { Concentração } \\
\text { inibitória de } \\
\mathbf{5 0 \%} \text { de DPPH }\end{array}$ \\
\hline 1- Extrato hexano- folha seca & 67,40 & 32,60 & $1062,52 \mu \mathrm{\mu g} / \mathrm{mL}$ \\
2- Extrato alcoólico- folha seca & 67,47 & 30,27 & $959,09 \mu \mathrm{g} / \mathrm{mL}$ \\
3- Extrato aquoso- folha seca & 21,02 & 78,83 & $370,9 \mu \mathrm{\mu g} / \mathrm{mL}$ \\
4- Extrato alcoólico- folha fresca & 86,73 & 13,27 & $2709,2 \mu \mathrm{g} / \mathrm{mL}$ \\
5- Extrato aquoso- folha fresca & 84,89 & 14,62 & $2237,0 \mu \mathrm{g} / \mathrm{mL}$ \\
6- Infusão alcoólica & 86,92 & 13,08 & $2173,67 \mu \mathrm{\mu g} / \mathrm{mL}$ \\
7- Infusão aquosa & 75,62 & 24,38 & $1280,81 \mu \mathrm{gg} / \mathrm{mL}$ \\
\hline
\end{tabular}

Tabela 3- Comparação do $\mathrm{CE}_{50}$ dos extratos alcoólicos, aquosos e hexanolicos de Ruta graveolens e de folhas de diferentes espécies.

\begin{tabular}{l|c|c|c}
\hline Espécie & $\begin{array}{l}\mathrm{CE}_{50}(\boldsymbol{\mu g} / \mathbf{~ m L}) \\
\text { Extrato } \\
\text { Alcoólico }\end{array}$ & $\begin{array}{l}\mathbf{C E}_{50}(\boldsymbol{\mu g} / \mathbf{~ m L}) \\
\text { Extrato } \\
\text { Aquoso }\end{array}$ & $\begin{array}{l}\mathbf{C E}_{50}(\boldsymbol{\mu g} / \mathbf{~ m L}) \\
\text { Extrato } \\
\text { Hexanólico }\end{array}$ \\
\hline Pseudobombax (LIMA, 2010) & 108,2 & 833,3 & \\
Eugenia uniflora L (VICTORIA et al, 2011) & 23,4 & 35,2 & 134,3 \\
S. paniculatum (RIBEIRO et.al, 2007) & 48,9 & & \\
Piper aleyreanum (SILVA et al, 2009) & 44,8 & & \\
Piper marginatum (SILVA et al, 2009) & 330,6 & & 73,1 \\
Piper dilatatum (SILVA et al, 2009) & & 38,9 & \\
Mimosa paraibana (NUNES et al, 2008) & & 2237 & \\
Ginkgo biloba (VICENTINO; MENEZES, 2007) & 2709,2 & 370,9 & 1139,3 \\
Ruta graveolens- Folha Fresca & 959,1 & & \\
Ruta graveolens- Folha Seca & &
\end{tabular}

PERIÓDICO TCHÊ QUÍMICA • www.periodico.tchequimica.com • Vol. 12 N. 23

• ISSN 1806-0374 (impresso) • ISSN 1806-9827 (CD-ROM) • ISSN 2179-0302 (meio eletrônico)

(C) 2015. Porto Alegre, RS. Brasil

The Periódico Tchê Química (ISSN: 1806-0374; 2179-0302) is an open-access journal since 2004. Journal DOI: 10.52571/PTQ. http://www.tchequimica.com. This text was introduced in this file in 2021 for compliance reasons.

() The Author(s)

OPEN ACCESS. This article is licensed under a Creative Commons Attribution 4.0 (CC BY 4.0) International License, which permits use, sharing, adaptation, distribution, and reproduction in any medium or format, as long as you give appropriate credit to the original author(s) and the source, provide a link to the Creative Commons license, and indicate if changes were made. The images or other third-party material in this article are included in the or exceeds the permitted use, you will need to obtain permission directly from the copyright holder. To view a copy of this license, visit http://creativecommons. 